\title{
Hacia un nuevo Estado desarrollista: desafíos para América Latina
}

\author{
Cristina Zurbriggen, ${ }^{*}$ Emiliano Travieso**
}

Perfiles Latinoamericanos, $24(47)$

2016 | pp. 259-281

DOI: $10.18504 / \mathrm{pl} 2447-004-2016$

\begin{abstract}
Resumen
La primera década del siglo xxI constituyó una coyuntura de transformaciones en el mapa político de América Latina. Después de una larga hegemonía de las fuerzas políticas de derecha y centro-derecha, las de izquierda y centro-izquierda — un conjunto muy heterogéneo- conquistaron los gobiernos de la región con un discurso crítico del modelo neoliberal, que subrayaba el papel del Estado como actor clave del proceso de desarrollo. En este escenario, la crisis de 2008 profundizó este debate, enfocando la relación del Estado con las élites y el capital extranjero, así como en su capacidad de articular una estrategia de desarrollo para construir sociedades más prósperas y justas. Este artículo revisita los dos paradigmas del papel del Estado en los procesos de desarrollo: el modelo neoliberal y el desarrollista, a la luz de enfoques teóricos de disciplinas como la ciencia política, la historia económica y la economía del desarrollo. Se concluye que el gran desafío de una nueva teoría y práctica del Estado en América Latina se encuentra en construir coaliciones desarrollistas amplias que permitan contrapesar los intereses centrífugos de las élites locales y las corporaciones trasnacionales.
\end{abstract}

\begin{abstract}
The political map of Latin America experienced some significant changes during the first decade of the $21^{\text {st }}$ century. After a long period of political and social hegemony of right-wing coalitions, progressive parties assumed office in many countries. Their programs criticised the neoliberal model and underlined the role of the State in the development process. In that context, the 2008 world economic crisis strengthened the debate on the role of the State, particularly in what regards to its rapport with elites and foreign capital, as well as its capacity to lead a development strategy towards more prosperous and just societies. This article revisits two theoretical paradigms (the Neoliberal State and the Developmental State) in the light of new approaches from different disciplinary grounds (Political Science, Economic History, Political Economy of Development). We argue the greatest challenge of a new theory and practice of the State in Latin America is to build broad developmental coalitions that are able to counter the centrifugal interests of local elites and transnational corporations.
\end{abstract}

Palabras clave: Estado desarrollista, América Latina, desarrollo económico, capacidades estatales, élites, corporaciones trasnacionales.

Keywords: Developmental State, Latin America, economic development, State capacities, elites, transnational corporations.

* Instituto de Ciencia Política, Facultad de Ciencias Sociales, Universidad de la República (Uruguay).

** Programa de Historia Económica y Social, Facultad de Ciencias Sociales, Universidad de la República (Uruguay). 


\section{Introducción}

N

uevas ideas, prácticas, políticas y enfoques de las teorías del desarrollo están surgiendo en la discusión sobre la necesidad de repensar el papel del Estado en el siglo Xxi. En este escenario, América Latina asiste a un "retorno del Estado" asociado a gobiernos de izquierda y centro-izquierda, cuyo discurso presenta claras innovaciones, como un rechazo categórico al modelo neoliberal, aspiraciones nacionales de crecimiento centradas en el bienestar y la ciudadanía, así como el fortalecimiento de la capacidad estatal para alcanzar un desarrollo nacional, propio o endógeno (Grugel \& Riggirozzi, 2012).

En este proceso, la crisis financiera mundial de 2008 profundizó aún más el escepticismo a nivel global hacia las principales recomendaciones de política "más mercado y menos Estado", y abrió el camino a la reconsideración del papel de este último en los procesos de desarrollo, replanteando las preguntas que parecían haberse cerrado en la década de los noventa (Wade, 2010; Stubbs, 2011; UNCTAD, 2011).

Estos debates plantean distintas interrogantes: ¿cuál es el futuro del Estado?, ¿un Estado desarrollista?, ¿hay espacio para un modelo de ese tipo en estos tiempos de creciente globalización y desregulación financiera?, ¿qué espacio hay para esa alternativa en América Latina?

Diferentes teorías y orientaciones políticas e ideológicas han identificado qué tipo de Estado y qué arreglos institucionales promovían su desarrollo en el siglo xx. Investigaciones previas realizadas por Johnson (1982), Evans (1995), Wade (1990) y Amsden (1989, 2001, 2003); Amsden \& Hikin (1994) han señalado la relevancia del Estado desarrollista y han demostrado la importancia de un servicio civil weberiano y la relevancia de algún organismo clave responsable de conducir estratégicamente una política de largo plazo en pro del desarrollo.

En la actualidad numerosas investigaciones en economía y ciencia política abogan por la importancia de la capacidad del Estado para la prosperidad económica y el desarrollo social. Sin embargo, parece que todavía tenemos que explicar la esencia de ese desafío y qué tipo de reformas se deben adoptar. Como señala Ostrom (2010), es tiempo de dejar atrás la dicotomía simplista entre Estado y mercado, y construir un marco alternativo que permita identificar los elementos y las características del nuevo Estado desarrollista, considerando el cambio de paradigma que actualmente ocurre.

Con este objetivo, en primer término, se analizan los dos paradigmas ordenadores de la discusión sobre el papel del Estado en los procesos de desarrollo (el modelo neoliberal y el modelo desarrollista), a la luz de nuevos enfoques 
teóricos provenientes de diversas disciplinas. En segundo, se sugieren las lecciones que debemos considerar si queremos construir una nueva teoría y práctica del Estado en América Latina, a fin de fortalecer la discusión sobre su rol para lograr un desarrollo sostenible (económico, social, ambiental y cultural) en el nuevo contexto histórico.

\section{Estado y desarrollo: dos paradigmas para ordenar la discusión}

\section{El Estado desarrollista asiático: una relectura desde el siglo XXI}

En las últimas tres décadas, la discusión sobre el papel del Estado en el proceso de desarrollo se ha bifurcado en dos paradigmas. Por un lado, el neoliberal que se ha constituido en la visión dominante sobre la estrategia económica, tanto en los principales Estados occidentales (sobre todo, Estados Unidos y Gran Bretańa) como en las instituciones financieras internacionales como el Banco Mundial (вм) y el Fondo Monetario Internacional (FмI) (Wade, 2010, pp. 2-3; Stubbs, 2011). Por otro lado, el paradigma del modelo de Estado desarrollista que se basó en las experiencias de los países del sudeste asiático.

La literatura sobre el Estado desarrollista ${ }^{1}$ ha intentado explicar el rápido crecimiento de los países del sudeste asiático en los años sesenta y setenta, y discutir si es posible encontrar un cierto grado de consenso en lo que se refiere al Estado como piedra angular de este notable éxito económico (Amsden, 1989; Johnson, 1982; Evans, 1995; Woo-Cummings, 1999; Wade, 1990; Leftwich, 1995; Chang, 1999). Los Estados desarrollistas han sido caracterizados como Estados con capacidad estratégica que lograron definir un modelo de inserción internacional, con base en un fuerte consenso político (crecimiento económico considerado como una prioridad de interés nacional), a través de las agencias del gobierno (o un organismo clave), y con una burocracia de extracción meritocrática altamente cualificada. Estas agencias tuvieron la capacidad de coordinar y conducir los esfuerzos de política a largo plazo hacia el desarrollo, con gran acierto para establecer alianzas con los sectores productivos por medio de redes formales e informales (consejos deliberativos, grupos de estudios de políticas, etc.). Asimismo, estos espacios facilitaron información relevante y procesos de aprendizaje interactivo para elaborar e implementar políticas ajustadas a los contextos nacionales (Amsden, 1989; Wade, 1990; Chang, 1999).

1 La noción de Estado desarrollista fue introducida por Johnson en 1982 cuando analiza a Japón como el precursor clásico, seguido de los cuatro "tigres" (República de Corea, Taiwán, Singapur y Hong Kong) en los años sesenta y setenta.

C. Zurbriggen, E. Travieso | Hacia un nuevo Estado desarrollista: desafíos para América Latina Perfiles Latinoamericanos, 24(47) | FLACso México | pp. 259-281 | DoI: 10.18504/pl2447-004-2016 
Por su parte, Chang (2012) resalta la flexibilidad del Estado que permitió poner en práctica diferentes políticas y retransformarse tan pronto como fuera posible ante un cambio de las condiciones del contexto. Por ello resalta la capacidad de estos países de integrar políticas de educación con políticas industriales y comerciales; efectivos subsidios al sector privado en $\mathrm{I}+\mathrm{D}$; inversiones públicas en áreas centrales; regulación de licencias tecnológicas, y regulación de inversiones extranjeras para maximizar en tecnologías.

Por eso también importa tener en cuenta otras lecciones menos conocidas sobre el Estado desarrollista asiático. Suele hacerse - no sin buenos motivosmucho hincapié en la política industrial, pero no se ha destacado igual el papel de las políticas sociales y el proceso de reducción de las desigualdades. En otras palabras, como señala Evans (2007), la fascinación con la industrialización ha distraído la atención sobre la trascendencia de la expansión de la capacidad humana en el éxito de los países del sudeste asiático. Éstos fueron pioneros en la inversión en capital humano y el éxito inicial del desarrollo económico tuvo una relación estrecha con la movilidad social creada por la reforma agraria (Kwon, 2005; Kay, 2002). Por otra parte, aunque esos gobiernos introdujeron con un alcance mínimo los programas de política social, fueron capaces de reducir la pobreza de modo efectivo y en etapas sucesivas. Es así que, mediante la movilización y la coordinación de las diversas instituciones responsables de las políticas económicas y las sociales, se logró reducir la pobreza y ampliar la protección social. La política social resultó fundamental en la estrategia general de desarrollo económico.

Diversos estudios plantean que la prosperidad económica y la seguridad social no tienen por qué ser antagónicos, o ideales mutuamente excluyentes. Diversos autores sugieren que los países del sudeste asiáticos lograron mantener el bienestar social al conseguir un diseño de políticas sociales articuladas a un patrón de acumulación basado en una economía del conocimiento en un contexto globalizado. La inversión en capital humano a través de la educación superior y continua, la capacitación laboral y otros programas para reincoporar al trabajo a los desempleados se recupera con creces mediante la reducción del gasto en prestaciones por desempleo y costos sociales (Kim \& Lee, 2010).

Así, un volumen creciente de estudios asiáticos cuestiona el paradigma productivista/desarrollista que ha tipificado a los Estados de bienestar en Asia, en el cual la política social sólo existe para asistir al desarrollo económico. Por el contrario, la relación entre el desarrollo económico y la política social ya no ha sido de subordinación, sino más bien simbiótica (Ringen et al. 2011; Hwang, 2012; Kim \& Lee, 2010).

Por su parte, otro aporte relevante en debate sobre capacidad de los Estados desarrollistas en un contexto de globalizacion y desregulación financiera es 
realizado por Amsden (2013), quien afirma que el rasgo más sobresaliente del Estado desarrollista del xxi es su carácter nacionalista, y su rechazo del universalismo de libre mercado. Un elemento clave de esta clase de Estado es su capacidad para conducir, orientar, regular y gravar las inversiones extranjeras, lo que constituye un déficit en especial importante al analizar algunas experiencias recientes en América Latina.

\section{El Estado neoliberal}

La caída del Muro de Berlín, el colapso de la Unión Soviética y los límites del Estado de bienestar dieron paso al triunfo de una versión particular del capitalismo: la consolidación del modelo de desarrollo neoliberal. De esta manera, el mercado parecía ser el único mecanismo con capacidad de canalizar los diferentes intereses dentro de una sociedad con el fin de garantizar el crecimiento económico y la movilidad social. Es así que, a principios de los noventa, este nuevo modelo de desarrollo centrado en el mercado fue auspiciado por los organismo multilaterales (en particular por el Fondo Monetario Internacional y el Banco Mundial) y sintetizado en el Consenso de Washington (Williamson, 1990), lo que incluyó una serie de reformas implementadas ampliamente en diversos contextos institucionales y económicos, tanto en América Latina como en Europa del Este y Asia Oriental: estabilidad macroeconómica, liberalización financiera y comercial, apertura de las economías a la inversión extranjera, privatización, desregulación, y concesiones a las empresas multinacionales.

A mediados de la década del noventa, sin embargo, se produjo un punto de inflexión en el debate internacional. El pobre desempeño económico de los países en desarrollo y, especialmente de América Latina, con altos niveles de pobreza y desigualdad acentuados por el modelo neoliberal condujo al replanteamiento del papel del Estado. Los estudios de Peters Evans y, sobre todo, del propio BM, apoyado por los aportes de su entonces vicepresidente, José Stiglitz (1999), plantearon la importancia de fortalecer las capacidades estatales para edificar una robusta economía de mercado, reconociendo como antecedente el milagro del sudeste asiático.

En este contexto, el informe del Banco Mundial de 1997, El Estado en un mundo en cambio, ${ }^{2}$ marcó un hito cuando se planteó la reforma del Estado como

2 La elaboración del informe generó una intensa discusión en el seno del propio organismo. Por un lado, aquellos que defendían la necesidad de una nueva generación de reformas superadora del Consenso de Washington (posición liderada por Stiglitz), que le otorgara una mayor capacidad de regulación al Estado en los sectores donde el mercado no puede asegurar el desarrollo. Por otro, aquellos que sostenían 
un elemento clave en la nueva agenda para el desarrollo. Ese informe destacó el carácter vital de las instituciones gubernamentales y la estabilidad política para lograr el desarrollo económico en el largo plazo y, en particular, para emprender una regulación satisfactoria de los mercados. Se planteaba la necesidad de un Estado eficaz para proporcionar los bienes y servicios - y las normas e instituciones- que permiten que los mercados prosperen y mejoren el nivel de vida. En consecuencia, en la nueva perspectiva del Banco Mundial, el Estado es fundamental para el desarrollo económico y social, no como proveedor directo de servicios, sino como un socio, catalizador y facilitador de las fuerzas del mercado (Zurbriggen, 2007).

En resumen, este consenso pos-Washington resaltó la importancia de las instituciones para el desarrollo económico, impulsando a los gobiernos a concentrarse en las tareas públicas básicas, aquellas que ni los mercados ni la sociedad civil pueden llevar a cabo: el establecimiento de un orden jurídico necesario, el mantenimiento de un entorno eficaz de políticas macroeconómicas e instituciones financieras, la inversión en servicios sociales básicos e infraestructura, el suministro de una red de seguridad integral para los miembros vulnerables de la sociedad y la protección del ambiente.

Así, este giro dio un paso adelante significativo en comparación con los modelos neoliberales ortodoxos, al reconocer el papel explícito de las instituciones en el desarrollo económico. Sin embargo, este modelo de Estado es muy diferente al Estado desarrollista, la intervención del Estado se reduce a otorgar mayor capacidad reguladora donde el mercado no puede llegar, al mismo tiempo que limitar la corrupción y paliar situaciones de pobreza extrema.

\section{Resiliencia del Estado neoliberal: coaliciones desarrollistas y neoliberales a nivel global}

La crisis financiera de 2008 significó un duro golpe al paradigma neoliberal en el debate global sobre el desarrollo. Si bien en América Latina el escepticismo respecto al modelo centrado en el mercado ya era notable mucho antes, en el escenario internacional la crisis evidenció una vez más las consecuencias críticas de las dificultades de los gobiernos para subordinar una agenda de desa-

una línea más cercana a la agenda del Consenso de Washington (Burki/Perry). Terminó imponiéndose la primera, por lo que el informe del Banco Mundial colocó en el centro de su preocupación el desafío de la reconstrucción del Estado y sostuvo que las reformas institucionales constituyen un elemento central para el desempeńo de los países, más allá de las reformas económicas y la apertura externa. Este informe, así como todo el proceso de reformas de segunda generación, generó más consenso que las reformas de primera generación, tanto entre los diferentes sectores de la sociedad como en el sistema político. 
rrollo al capital financiero, lo que motivó algunas iniciativas de cambio en la gobernanza global.

Una expresión relevante fue que desapareció el G-7 como foro principal de coordinación de las potencias, y se le sustituyó con el G-20 —que incluye a países emergentes como Brasil, China e India, reconociéndolos como un nuevo grupo de actores políticos globales- En este contexto, se planteó el debate internacional sobre la política industrial y las políticas sociales, pero fundamentalmente sobre el retorno del Estado desarrollista (UCNTAD, 2011).

Desde luego, el diálogo político en el G-20 supone articular intereses diversos (antagónicos, en algunos casos) entre países muy diferentes (en términos de desarrollo relativo, estructuras productivas, capacidades estatales, etc.), en un contexto histórico en el que el poder de los Estados como tal aparece crecientemente cuestionado por otros actores (empresas transnacionales, capital financiero).

En este sentido, la lucha no es sólo acerca de los poderes económicos y políticos, sino también por la competencia global de ideas y modelos. Como señala Stubbs (2011), la gran recesión ha tenido un profundo efecto en el Este de Asia y en la batalla entre las coaliciones desarrollistas y neoliberales. Significó un duro golpe a la reputación de Estados Unidos como modelo a seguir (Krugman, 2010) y como centro principal de producción de conocimiento y pensamiento innovador (Williamson, 2012). Al mismo tiempo, se han reforzado las coaliciones desarrollistas y, en particular, el modelo de crecimiento chino despierta una atención en aumento. ${ }^{3}$

De hecho, la crisis de 2008 no impactó de forma decisiva en la construcción de un nuevo marco regulatorio internacional, ni se tradujo en el final de la hegemonía del modelo neoliberal. Bermeo \& Pontusson (2012) argumentan sobre los límites de las respuestas multilaterales a la crisis de 2008, afirmando que las presiones de los principales actores económicos en cada país desarrollado fueron más fuertes que los planes de reforma y regulación financiera a nivel internacional. En la misma dirección, Schmidt \& Thatcher (2013) explican cómo el modelo neoliberal fue mutando, pero manteniendo sus principios esenciales, para continuar hegemonizando la teoría y la práctica del Estado en la Unión Europea y en sus principales países. Como señala Wade (2010), la retórica del modelo neoliberal todavía goza de buena salud en muchos ámbitos influyentes.

3 Particularmente, en América Latina es un tema de gran relevancia por la relevante presencia china en inversiones en la región y consumidora de recursos naturales. Para un estudio económico de los impactos del comercio y las inversiones chinas en la región véase Bittencourt (2012). Varios trabajos realizados en el ámbito de la CEPAL ofrecen análisis de balance y perspectivas de la relación China-América Latina desde la preocupación por el desarrollo latinoamericano: Rosales (2013), NU/CEPAL. División de Desarrollo Productivo y Empresarial (2012), un/CEPAL. División de Comercio Internacional e Integración (2012). 
En definitiva, la complejidad del escenario actual implica el desafío de superar los planteos dicotómicos "mercado versus Estado" y generar nuevos debates que permitan comprender qué tipos de capacidades estatales y coaliciones reformistas son necesarias para un desarrollo sostenible e inclusivo en el siglo xxI.

\section{Nuevas miradas sobre viejos problemas. ¿Hacia un nuevo Estado desarrollista?}

Un elemento común de las nuevas ideas, teorías, prácticas, políticas y enfoques para el desarrollo es el énfasis en la necesidad de repensar el papel del Estado. Los nuevos enfoques plantean sugerentes debates en torno al cambio del rol del Estado: promover el capital humano y el medio ambiente, fortalecer una lógica distributiva equitativa de los ingresos o fortalecer las instituciones democráticas deliberativas como factores claves para el proceso de desarrollo. ${ }^{4}$ Estas ideas se pueden encontrar en la nueva literatura de la economía del desarrollo, o en nuevos enfoques de desarrollo y los Estados desarrollistas, y en los modelos de Estado de bienestar en un contexto de globalización y desregulación del capital financiero. Sin embargo, estas comunidades epistémicas realizan importantes aportes a la comprensión del fenómeno, pero no dialogan con fluidez entre sí.

\section{El Estado desarrollista en el siglo XXI}

Desde nuevos enfoque de las teorías de desarrollo y del Estado desarrollista se plantea que la discusión actual sobre crecimiento económico, y los trabajos empíricos en ese terreno, con base filosófica en el enfoque de Sen, subrayan el importante papel del capital humano como motor de la dinámica de crecimiento de las economías y la necesidad de desarrollar políticas públicas (Evans \& Heller, 2013).

En esta dirección, un Estado desarrollista - también en el contexto histórico del siglo XXI- debe avanzar en fortalecer las capacidades para impulsar el

4 En este apartado se plantean sólo alguno de los debates recientes en torno a repensar un nuevo rol del Estado para un nuevo desarrollo, lo que es una forma de comenzar a generar una nueva línea de investigación, en la que se podrán incorporar nuevos marcos analíticos en un futuro. Sólo a modo de ejemplo, se han producido otros debates en torno a los libros de Piketty (2014) o Mazzucatto (2013). También ha tenido lugar una discusión política en Europa respecto a la contribución original de Ostrom (1991) sobre los bienes comunes y los nuevos aportes. 
capital humano y aumentar la oferta de bienes públicos, como la educación y salud. Estos objetivos, a su vez, muestran la importancia de la política social y de bienestar para el desarrollo y la necesidad de nuevos y ampliados vínculos entre Estado y sociedad para el éxito de aquél.

La eficacia de las estructuras administrativas depende cada vez más de las nuevas formas de arraigo democrático. Por lo tanto, hay ciertas formas democráticas de arraigo que tienen más probabilidades de fortalecer la intervención del Estado para mejorar las capacidades (Evans \& Heller, 2013). En este sentido, uno de los elementos esenciales del Estado desarrollista es su poder de autoridad, entendido como "[...] the power to get individuals and groups to willing obey commands" (Evans \& Heller, 2013: p. 8). Estrechamente relacionado con aquél se encuentra el poder infraestructural, entendido como la capacidad de proporcionarle a la sociedad los bienes y servicios que exige (Evans, 2013). La capacidad del Estado de cumplir con estos requerimientos, le otorga legitimidad, con lo que aumenta su poder de autoridad y genera así un círculo virtuoso donde un mayor poder de autoridad le permite tomar las decisiones pertinentes para proporcionar mayor poder infraestructural.

Una expresión de la capacidad autoritativa es la capacidad fiscal, clave no sólo para sostener las capacidades del Estado sino para cumplir con los objetivos de redistribución del ingreso y equidad.

Esta transformación del Estado se encuentra condicionada por nuevos factores históricos. Los ciudadanos demandan mejores y más personalizadas soluciones y servicios públicos, pero éstos no pueden ser satisfechos con el simple gasto de más dinero público. En segundo lugar, hay un número creciente de problemas complejos en términos de reducción de la pobreza, seguridad pública, planificación urbana, medio ambiente, entre otros. La resolución de éstos requiere la articulación de diferentes conocimientos y la participación de distintos actores, a través de un proceso continuo de experimentación; Sabel \& Zeitlin (2012) señalan que eso intensifica las interacciones entre Estado y sociedad civil, las que son fundamentales para la innovación política y los avances hacia una democracia deliberativa (Sabel, 2012).

Los gobiernos están adoptando una colaboración más estrecha con los ciudadanos. De hecho, puede pensarse a la educación como un servicio coproducido por los estudiantes (y sus familias). La salud es coproducida por los pacientes, sus familias y sus comunidades o la protección del medio ambiente por las comunidades (Ostrom, 1996). La política de innovación es coproducida entre el sector privado y la sociedad civil (Baiocchi, Heller \& Silva, 2011). El Estado desarrollista del siglo XXI necesita la construcción de objetivos coherentes, cambiantes y compartidos, cuya aplicación concreta debe resultar del esfuerzo conjunto de los organismos públicos y las propias comunidades. 
De esto se deduce que la política pública tiene que ser lo suficientemente flexible para permitir una variedad de esfuerzos y marcos regulatorios. También debe ser lo suficientemente revisable para que los frutos del aprendizaje se puedan incorporar con facilidad. Por último, se requiere de formas abiertas y transparentes de gobierno, sin lo cual las nuevas ideas no se filtran hacia arriba ni pueden ser ampliamente compartidas. En ese sentido, las instituciones deliberativas son factores clave para el desarrollo. En resumen, como apuntan Evans \& Heller (2013: p. 10), la transformación del Estado implica dirigir la atención analítica a las conexiones entre el aparato estatal y el conjunto de los actores de la sociedad civil, en lugar de privilegiar los vínculos con un grupo restringido de aliados de élite.

\section{Acumulación y distribución de ingresos}

Desde los enfoques económicos se hace fuerte énfasis en los aspectos distributivos. Es así que otros debates han subrayado la importancia de una distribución relativamente equitativa de los ingresos para el crecimiento económico (Alesina \& Rodrik, 1994; Bardhan \& Ghatak, 1999; Engerman \& Sokoloff, 2002; Sokoloff $\&$ Zolt, 2006). La línea argumental de tales debates sugiere que la inequidad, al concentrar los recursos políticos y económicos en manos de una minoría, limita la inversión en bienes públicos e infraestructura y afecta negativamente las posibilidades de cooperación. Engerman \& Sokoloff (2002) trabajan con esta idea como herramienta para la interpretación histórica, argumentando que la consolidación de élites reducidas en América Latina a lo largo de los tres siglos posteriores a la conquista europea, a través de un marco institucional concentrador (política de tierras, lógicas de participación política y cobertura educativa), constituye un factor clave para entender la trayectoria divergente con relación a América del Norte. El análisis de los sistemas impositivos en perspectiva histórica también enfatiza la relevancia de cierta equidad en la distribución de la renta para el desarrollo económico en el largo plazo. Sokoloff \& Zolt (2006) afirman que la desigualdad inicial en términos de ingreso, tierra y participación política afecta el desarrollo de las instituciones impositivas, que hacen persistentes los escenarios de inequidad, constituyen un límite al gasto público e influyen en el crecimiento económico. Este mecanismo puede leerse como una expresión de la llamada "paradoja de Robin Hood" (Lindert, 2004: p. 15), la cual señala que las políticas redistributivas están más presentes cuando son menos necesarias.

Por su parte, Alice Amsden subrayó la misma idea central: "The more the distribution of the incomes is equal [...] the faster will be the growth rate of industrial productivity" (Amsden, 1992: p. 78). Existe también un argumento 
de economía política relevante en esa relación: una distribución inequitativa origina una élite poderosa que puede dificultar la independencia de las políticas públicas y la capacidad del Estado (Amsden \& Hikino, 1994).

Estos cambios en la teoría y la práctica conducen a la necesidad de repensar el rol del Estado y las políticas de desarrollo en América Latina. Se debe de refinar la teoría por medio de un mayor diálogo entre comunidades epistémicas y realizar mucho más trabajo empírico para determinar el grado en que esta nueva orientación se está consolidando en todo el mundo.

\section{Los desafíos del desarrollo en América Latina y las capacidades del Estado}

Los descontentos políticos y los grupos sociales excluidos por el modelo de desarrollo neoliberal posibilitaron que, después de una larga hegemonía de las fuerzas de derecha y centro-derecha, las de izquierda hayan conquistado el poder. La tendencia comenzó en 1998 con el triunfo en Venezuela de Hugo Chávez, luego fue Ricardo Lagos en Chile en 2000 y Luiz Inácio Lula da Silva en Brasil en 2002. Siguieron Argentina, Bolivia, Brasil (nuevamente), Chile, Costa Rica, Guatemala, Ecuador, Nicaragua, Paraguay y Uruguay (Panizza, 2009). ${ }^{5}$

Estas fuerzas políticas de centroizquierda altamente heterogéneas tienen en común un fuerte rechazo por el modelo neoliberal y la necesidad de impulsar un nuevo modelo de desarrollo. En este proceso, el Estado es un actor clave y, por tanto, la región asiste a un "retorno del Estado" asociado — para algunos autores - al neoestructuralismo (CEPAL, 2007) o para otros al posneoliberalismo (Grugel \& Riggirozzi, 2007, 2009; Hershberg \& Rosen, 2006; Roberts, 2008; Ruckert \& MacDonald, 2010). Así, el ingreso al siglo xxi coincide con el agotamiento del neoliberalismo ortodoxo y la irrupción de nuevos gobiernos caracterizados por su rechazo de las políticas neoliberales y su defensa de una mayor intervención del Estado (Boschi \& Gaitán, 2009).

Sin embargo, después de una década en el gobierno, han aparecido importantes debates en la región que plantean que estas nuevas fuerzas políticas han sido incapaces de construir un modelo de desarrollo alternativo. Y han generado fuertes críticas por su alta dependencia de los commodites, su vulnerabilidad y sus políticas focalizadas que se supeditan al crecimiento económico y a las lógicas del mercado (Panizza, 2009; Leiva, 2008; Filgueria, 2009; Boschi \& Gaitán, 2009). Estas últimas, por otra parte, no se han mostrado eficientes en la distribución de

5 Sobre la discusión posneoliberal en América Latina desde la ciencia política véase también Burdick, Oxhorn \& Roberts (2009), Leiva (2008) y Silva (2009). 
los ingresos, de tal modo que el desempleo, el subempleo y la subutilización de los recursos han empeorado, agudizando la dualidad de la región.

Filgueira (2009) es más crítico y afirma que los niveles y las formas de desigualdad actuales en América Latina son incompatibles con una estrategia de desarrollo, pues un Estado social se sustenta en la regulación del capital internacional y en los pactos fiscales. Ambos aspectos son posibles sólo en Estados fuertes que puedan implementar tales medidas. En cuanto a la regulación del gran capital, un Estado fuerte permite tomar las medidas necesarias para gravar y controlar al gran capital transnacional y a las élites nacionales. Pero en América Latina el Estado es muy débil en ambos aspectos.

Por su parte, la CEPAL — con una versión actual más crítica y menos matizada que la de principios de los noventa - plantea que los desafíos del desarrollo latinoamericano han estado históricamente asociados a las dificultades para superar los rasgos de heterogeneidad de la estructura productiva, los cuales constituyen la base de los problemas distributivos característicos de la región. Si bien en los últimos diez años el desempeño económico, impulsado por el sector externo, ha permitido niveles de crecimiento superiores a la media mundial, la tendencia de largo plazo a divergir respecto de los niveles de ingreso per cápita de los países desarrollados no se ha modificado sustantivamente. Además, la volatilidad propia de las economías latinoamericanas continúa condicionando fuertemente las expectativas de los actores en términos de inversiones a mediano y largo plazos. El gran desafío consiste en fortalecer la capacidad de los Estados latinoamericanos a fin de que construyan una economía política para el crecimiento y redistribución con base en la buena gobernanza de los recursos naturales de la región. Las propias inercias históricas de un modelo de desarrollo heterogéneo y volátil dificultan un proceso así.

La persistencia de un patrón de especialización productiva y comercial concentrado en bienes primarios (en el caso de América del Sur) y en manufacturas de bajo y medio contenido tecnológico (México y América Central) plantea restricciones importantes a los esfuerzos de la política pública y condiciona el margen de acción del Estado, por lo menos a través de dos canales que nos interesa destacar. En primer término, retomando el argumento cepalino clásico, la especialización en bienes con baja elasticidad ingreso de la demanda genera recurrentes problemas de equilibrio externo, lo que dificulta la gestión macroeconómica de los gobiernos y afecta las posibilidades de sostener niveles de crecimiento del nivel de actividad. En otras palabras: cuando América Latina crece lo hace importando más de lo que exporta, lo que atenta contra el propio crecimiento (gráfico 1). En segundo lugar, las notables diferencias de productividad del trabajo entre las actividades asociadas al sector exportador y las destinadas al mercado interno se conservan como el punto de partida de los problemas distributivos de la región. 
Gráfico 1. América Latina: crecimiento del PIB y déficit de cuenta corriente (1990-2013)

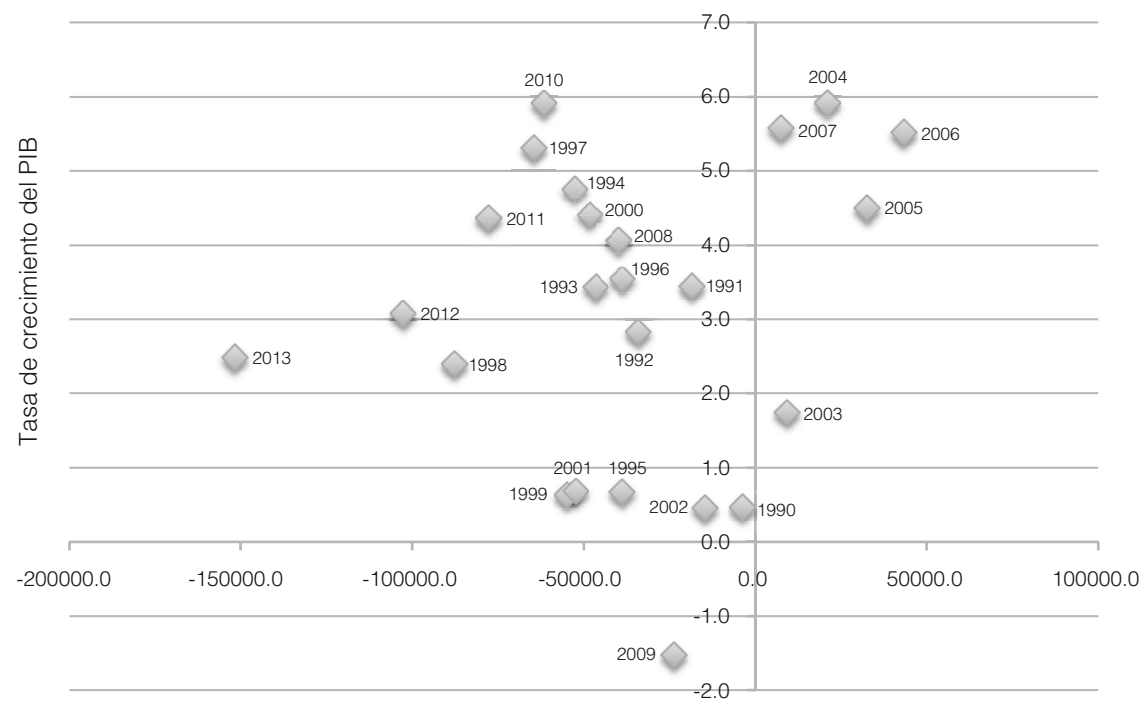

Balance de cuenta corriente

Fuente: Elaboración propia con base en CEPALSTAT.

Los destacados logros recientes en términos de reducción de pobreza y la mejora — más tímida — de los indicadores de desigualdad entre los hogares a partir de 2003, se asocian a una mayor presencia del Estado en el mercado de trabajo, lo que afecta en particular a los niveles de ingreso de los trabajadores más pobres. Las políticas de salario mínimo y formalización laboral se revelan como instrumentos limitados para avanzar en la reducción de las desigualdades, ya que sólo operan una mejora distributiva de baja intensidad a la interna de la masa salarial. La comparación en la evolución reciente del índice de Gini con la distribución funcional del ingreso entre retribuciones al trabajo y al capital muestra que el núcleo duro de la desigualdad latinoamericana no ha sido afectado (gráficos 2 y 3). En este sentido, los logros en el segundo momento de la fiscalidad (el gasto público) han encontrado límites fuertes no sólo en la heterogeneidad estructural sino también en el primer momento de la fiscalidad gobernado por estructuras tributarias de muy bajo potencial redistributivo. ${ }^{6}$

6 En promedio, en los países desarrollados de la ocDE, el índice de Gini cae unos quince puntos luego de los efectos de impuestos y transferencias. En América Latina, el efecto es muy menor, e incluso llega a ser regresivo en algunos países (Bárcena \& Serra, 2012: p. 82). 
Gráfico 2. Coeficiente de Gini en 2002 y 2012 para países seleccionados

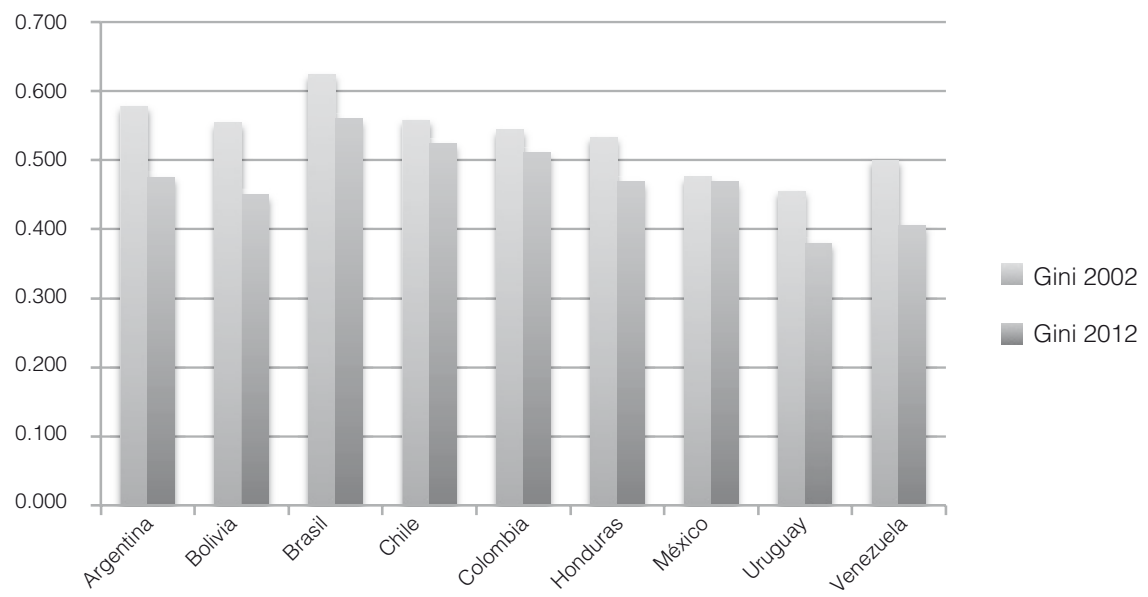

Fuente: CEPALSTAT.

Gráfico 3. Participación de la masa salarial en el ingreso (labor share) en 2000 y 2009

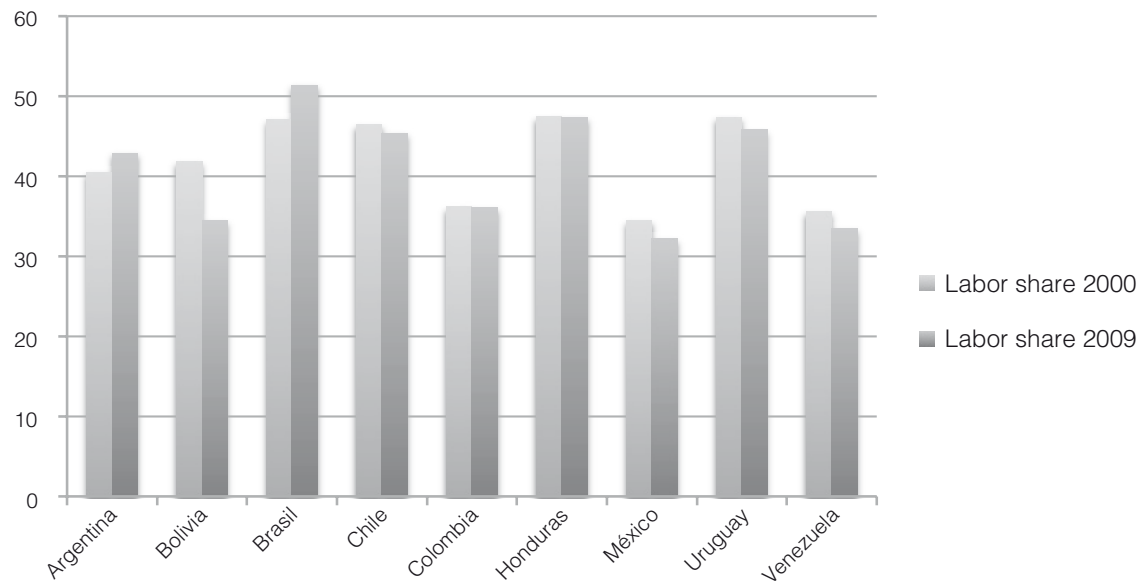

Fuente: Elaboración propia con base en datos de la CEPAL (2014: p. 77).

En definitiva, la inercia de una estructura productiva de baja densidad con una inserción internacional centrada en bienes de bajo dinamismo, y los resultantes desequilibrios externos ponen en riesgo la sustentabilidad económica de las mejoras del nivel de vida alcanzadas. Por otra parte, la persistencia de 
los núcleos duros de la desigualdad latinoamericana conspira contra la propia capacidad de los Estados para introducir cambios en la trayectoria, lo que genera un escenario de economía política donde la construcción de coaliciones y pactos para el desarrollo se hace difícil ante los intereses centrífugos de las élites y el capital extranjero.

\section{Una agenda futura de investigación}

En este escenario, volvemos a los interrogantes del inicio: ¿¿cuál es el futuro del Estado? ¿Un Estado desarrollista en el siglo Xxi? ¿Hay espacio para un modelo de ese tipo en estos tiempos de creciente globalización y desregulación financiera? ¿Qué espacio hay para esa alternativa en América Latina?

Para responder a esas preguntas es necesario un análisis de cómo las transformaciones profundas del escenario internacional, del modelo de desarrollo y los cambios en la economía y la sociedad han impactado en los Estados nacionales. En la región ha faltado un análisis más integrado entre factores políticos, económicos y sociales como parte de la agenda de la democracia. Han sido muy limitados los avances en comprender la relación entre Estado, régimen democrático, transformación económica y demandas sociales, lo que vincula al régimen político con el Estado y, en esta línea, con la preocupación por la calidad democrática y con el ejercicio del poder en un nuevo modelo de desarrollo. En otras palabras, en comprender la relación entre regímenes de producción y de bienestar, capacidades estatales y relaciones de poder.

La complejidad del escenario actual implica un gran desafío para superar los planteos dicotómicos "mercado versus Estado" y generar nuevos marcos analíticos que permitan comprender qué tipo de Estado requerimos para un desarrollo sostenible e inclusivo en el siglo xxi. Más allá de indicadores que muestren los resultados de las políticas y nos permitan comparaciones internacionales, necesitamos avanzar en los estudios para comprender las restricciones que impiden la construcción de las sinergias que lleven adelante las transformaciones a fin de avanzar hacia un nuevo modelo de desarrollo.

En los recientes debates sobre los Estados desarrollistas en el siglo XXI, se plantea el papel central que debe desempeñar el Estado fortaleciendo las capacidades para generar capital humano. Sin embargo, estas capacidades no se expresan sólo en políticas públicas específicas, sino también en los problemas cruciales de cómo el Estado regula al capital internacional y la distribución de la riqueza. Una distribución inequitativa genera una élite poderosa que puede dificultar la independencia de las políticas públicas y la capacidad del Estado (Amsden \& Hikino, 1994). 
Así, el desarrollo económico debe ser visto como parte del proyecto de construcción nacional (Gerschenkron, 1968). Un proceso de transformación productiva no se debe concentrar sólo en políticas de innovación o de educación, sino también en las condiciones sistémicas, fundamentalmente en las relaciones de poder entre capital y Estado y las desigualdades que impactan en los procesos democráticos de toma de decisión.

Como señala Chang (2003), el accionar público es necesario, pero además debe ser eficiente y depende en buena medida de su posibilidad de alterar relaciones de poder. Las características y el comportamiento de las élites económicas son centrales en ese sentido. Por ello, Amsden (2001) señala que al momento del despegue coreano (principios de la década de 1960), la distribución del ingreso era mucho más igualitaria que en América Latina. En consecuencia, las élites eran significativamente más débiles y pudieron ser disciplinadas desde un Estado que otorgó subsidios a la producción industrial y protección de mercado a cambio de metas de desempeño. En otros términos, el Estado logró redistribuir recursos y que las transferencias de ingresos a la élite derivaran en que ésta se volviera innovadora y eficiente, bajo la amenaza de que, si no lo hacía, se la castigaría.

En América Latina, en cambio, las protecciones sin contraprestación e interrumpidas agresivamente por políticas liberales implicaron empresas demasiado orientadas a la búsqueda de ganancias fáciles y no basadas en el esfuerzo y la innovación. De este modo, el poder del Estado en relación a las élites y su capacidad de construir coaliciones en torno a una estrategia de desarrollo nacional se revela clave. Una dimensión importante en esa tarea es la micropolítica pública, tendiente a articular actores económicos y sociales relevantes para la agenda de transformaciones (Boschi \& Gaitán, 2013).

En este sentido, se debería avanzar hacia estudios comparativos como el reciente de Almeida \& Schneider (2012) sobre la relación del Estado y las élites en Brasil. Tanto en el discurso público como en la práctica política se enfatiza la innovación como un eje fundamental en las políticas industriales de los últimos. De hecho, Brasil ha desarrollado una serie de instrumentos así como muy fuerte apoyo desde el Banco Nacional do Desenvolvimento (BNDES). Sin embargo, estas políticas no han permitido cambiar la estructura productiva y la inserción internacional, y han mantenido la especialización exportadora en bienes de baja tecnología. Por su parte, estas políticas tampoco han impedido la fuerte concentración e internacionalización de las empresas. Dichos autores, asimismo, resaltan que a pesar de muchos cambios en la política y en el entorno económico y político, existe una continuidad significativa en las relaciones informales y poco transparente entre las empresas y el gobierno. Por lo tanto, no está claro en la política industrial actual si los políticos pueden recurrir a 
mecanismos para exigir reciprocidad y disciplina al capital, de manera de promover una política industrial exitosa como en los países del sudeste asiático. En consecuencia, la relación entre gobierno y élites condiciona fuertemente a la política industrial que tiende a apoyar a las grandes empresas existentes —en lugar de desarrollar nuevos sectores - y experimenta dificultades para controlar las dinámicas de extranjerización (Almeida \& Schneider, 2012).

En consecuencia, la relación de poder entre los Estados y las élites es un escollo importante para el desarrollo en América Latina, que requiere una agenda de investigación más abarcadora y comprensiva. Las corporaciones en la región cuentan con un poder de veto mucho mayor que en Asia. Muchas no son controladas por residentes locales y responden a estrategias globales que pueden no coincidir con los propósitos y necesidades nacionales de desarrollo. Es así que Amsden (2013) afirma que un Estado desarrollista en el siglo Xxi deber ser fuertemente nacionalista y regular el capital transnacional.

Al mismo tiempo, con los gobiernos progresistas han proliferado en América Latina una multiplicidad de experiencias de participación ciudadana que se han materializado en diversas iniciativas que han ampliado la voz de los ciudadanos y el horizonte de la democracia. Sin embargo, la calidad y el desempeño de la democracia es todavía muy desigual. Estas expresiones deberían ser investigadas como espacios de lucha y disputa donde se construye la relación entre Estado y ciudadanía, e indagar qué tipo de iniciativas democráticas pueden conducir a una redistribución del poder (Gurza \& Isunza, 2010).

Se impone profundizar en estudios comparativos que den cuenta de cómo las transformaciones latinoamericanas de las últimas décadas han generado grupos privilegiados que se han beneficiado con la estrategia (exportadores, importadores, productores y trabajadores de zonas francas, grupos profesionales y ejecutivos vinculados a las empresas transnacionales, etc.), y, como consecuencia, se han originado fuertes intereses a favor de la prolongación de la estrategia. Esto conduce a otro interrrogante sobre si la estrategia de desarrollo pro mercado y hacia afuera ha provocado cambios en las estructuras de poder locales; donde las empresas internacionales — y sus prácticas de vinculación local — han adquirido mucha mayor relevancia de la que tenían a fines de los años setenta (Puchet et al., 2012).

La liberalización económica global, la mayor independencia de los grupos de poder económico y la degradación de las instituciones públicas sufrida en las últimas dos décadas y media del siglo xx, así como la desigualdad en la distribución de la riqueza, son condicionantes importantes para el desarrollo de políticas en la región que precisan de más estudios en la región.

Con este foco más amplio se podría identificar los vínculos entre políticas públicas, instituciones del Estado, estrategias macroeconómicas y sociales y su 
contribución a un desarrollo que haga posible la emergencia un Estado que represente el interés de las mayorías. Un Estado que garantice los derechos económicos y sociales implica la capacidad de proveer servicios a todos los ciudadanos por igual y no servicios diferenciados que reproduzcan la desigualdad. Sin embargo, en América Latina, el Estado ha conducido a que los servicios se encuentren divididos en dos grupos: aquellos de asistencia dirigidos a las personas en situación de pobreza, y los encargados del bienestar de los estratos que tienen asegurado un adecuado nivel de integración social. En Latinoamérica no se genera una apropiación de los bienes colectivos, ya que a pesar de la universalidad declarada, hay sectores que por diferentes razones, no pueden usufructuarlos. Habría que indagar más si, en el plano económico-social, el modelo actual mantiene al mercado como único asignador de los recursos productivos y entrega de buena parte de los servicios de educación, transporte, salud, seguridad, etcétera.

Estas preguntas deberían ser un punto de partida para fortalecer una agenda de investigación en América Latina sobre el rol del Estado en las estrategias de desarrollo, y debatir sobre las fuertes tensiones no resueltas entre crecimiento, equidad y gobernabilidad, que son los tres componentes de la ecuación capitalismo (en tanto modo de producción), social (en tanto modo de redistribución equitativa del excedente) y democrático (en tanto modo de gobernabilidad) (Oszlak \& Gantman, 2007).

Estas discusiones reclaman una agenda de investigación centrada en la articulación de estructuras económicas y procesos sociopolíticos que traban la construcción de Estados capaces de impulsar procesos de desarrollo en América Latina. Frente a este desafío, la colaboración entre investigadores es fundamental para superar la excesiva compartimentación producida por la especialización y avanzar hacia estudios interdisciplinarios sobre el papel del Estado en los procesos de desarrollo.

\section{Referencias}

Alesina, A. \& Rodrik, D. (1994, mayo). Distributive Politics and Economic Growth. The Quarterly Journal of Economics, 109 (2), 465-490.

Almeida, M., Schneider, B. (2012, julio). Globalization, Democratization, and the Challenges of Industrial Policy in Brazil. Korean Development Institute.

Amsden, A. (2013). Securing the Home Market A New Approach to Korean Development. Research Paper 2013-1 unsRIS. 
Amsden, A. (2001). The Rise of the "Rest": Challenges to the West from Late-Industrializing Economies. Oxford University Press.

Amsden, A. (1989). Asia's Next Giant: South Korea and Late Industrialization. Oxford: Oxford University Press.

Amsden, A. \& Chu, W. W. (2003). Beyond Late Development: Taiwan's Upgrading Process. MIT Press.

Amsden, A. H. \& Hikino, T. (1994). Project execution capability, organizational know-how and conglomerate corporate growth in late industrialization. Industrial and Corporate Change, 3(1).

Baiocchi, G., Heller, P. \& Silva, M. (2011). Bootstrapping Democracy: Transforming Local Governance and Civil Society in Brazil. Stanford, California: Stanford University Press.

Banco Mundial (вм) (1997). Informe sobre desarrollo humano 1997. El Estado en un mundo en transformación. Washington, D.C.: Oxford University Press.

Bardhan, P. \& Ghatak, M. (1999). Inequality, Market Imperfections, and Collective Action Problems. Center for International and Development Economics Research (CIDER) Working Papers C99-108, University of California at Berkeley.

Bermeo, N. \& Pontusson, J. (2012, septiembre). Coping with Crisis: Government Reactions to the Great Recession. Nueva York: Russell Sage Foundation.

Bittencourt, G. (Coord.) (2012). El impacto de China en América Latina. Comercio e inversiones. Montevideo: Red Mercosur.

Boschi, R. \& Gaitán, F. (2013). Legados, política y consenso desarrollista. Transformación, Estado y Democracia. Transformación, Estado, Democracia, 8(52).

Burdick, J., Philip, O. \& Kenneth, M. R. (2009). Beyond Neoliberalism in Latin America? Societies and Politics at the Crossroads. Nueva York: Palgrave Macmillan.

Cameron, M. A. \& Sharpe, K. E. (2012). La voz institucionalizada en las democracias de América Latina. En Cameron, M. A., Hershberg, E. \& Sharpe K. E. (Eds.), Nuevas instituciones de democracia participativa en América Latina: la voz y sus consecuencias. México: Flacso México, 337-361.

CEPAL (2010). Balance preliminar de las economías de América Latina y el Caribe 2010. Santiago de Chile: CEPAL.

C. Zurbriggen, E. Travieso | Hacia un nuevo Estado desarrollista: desafíos para América Latina Perfiles Latinoamericanos, 24(47) | FLACso México | pp. 259-281 | DoI: 10.18504/pl2447-004-2016 
Chang, H. J. (2010). The East Asian Development Experience. En Chang, H. J. Rethinking Development Economics. Londres: Anthem Press.

Chang, H. J. (2003). Kicking away the ladder. Development strategy in historical perspective. Londres: Anthem Press.

Chang, H. J. (1999). The Economic Theory of the Developmental State. En Meredith, W. C. (Ed.). The Developmental State. Nueva York: Cornell University Press.

Engerman, S., Sokoloff, K. (2002). Factor Endowments, Inequality, and Paths of Development among New World Economies. NBER Working Paper núm. 9259.

Evans, P. (1995). Embedded Autonomy: States and Industrial Transformation. Princeton: Princeton University Press.

Evans, P. \& Heller, P. (2013). Human Development, State Transformation and the Politics of the Developmental State. Forthcoming.

Gerschenkron, A. (1968). El atraso económico en su perspectiva histórica. Barcelona: Ariel.

Gough, I. (2001). Globalization and Regional Welfare Regimes: The East Asian Case. Global Social Policy, 1(2), 163-189.

Gurza Lavalle, A., Isunza Vera, E. (2010). Precisiones conceptuales para el debate contemporáneo sobre la innovación democrática: participación, controles sociales y representación. En Isunza Vera, E. \& Gurza Lavalle, A. (Coords.). La innovación democrática en América Latina. Tramas y nudos de la representación, la participación y el control social. México: CIESAS/ Universidad Veracruzana.

Grugel, J. \& Riggirozzi, P. (2009). Governance after Neoliberalism in Latin America. Nueva York: Palgrave Macmillan.

Hershberg, E., Rosen, F., \& North American Congress on Latin America. (2006). Latin America After Neoliberalism: Turning the Tide in the 21st Century? Nueva York: New Press.

Hwang, G. J. (2012). Explaining Welfare State Adaptation in East Asia: The Cases of Japan, Korea and Taiwan. Asian Journal of Social Science, 40, 174-202.

Holliday, I. (2000). Productivist Welfare Capitalism: Social Policy in East Asia. Political Studies, 48(4), 706-23.

Johnson, Ch. A. (1982). MITI and the Japanese Miracle: The Growth of Industrial Policy, $1925-$ 1975. Stanford, C.A.: Stanford University Press. 
Kay, C. (2002, diciembre). Why East Asia Overtook Latin America: Agrarian Reform, Industrialisation and Development. Third World Quarterly, 23(6), 1073-1102

Kim, L. (1997). Imitation to innovation. The Dynamics of Korea's Technological Learning. Boston: Harvard Business School Press.

Kim, T. \& Lee, Kwon, (2010). Developments and General Features of National Health Insurance in Korea. Social Work in Public Health, 25(2), 142-215.

Krugman, P. (2010, 15 de octubre). The Mortgage Morass. New York Times, A33.

Kuhnle, S. \& Hort, S. (2004, septiembre). The Developmental Welfare State in Scandinavia.Lessons to the Developing World. Social Policy and Development Programme Paper núm. 17. UNRISD.

Kwon, H. J. (Ed.) (2005). Transforming the Developmental Welfare State in East Asia. Londres: Palgrave/Macmillan.

Leiva, F. I. (2008). Latin American Neostructuralism: The Contradictions of Post-Neoliberal Development. Mineápolis: University of Minnesota Press.

Lindert, P. (2004). Growing Public, vol. 1. The Story: Social Spending and Economic Growth since the Eighteenth Century. Cambridge: Cambridge University Press.

Mazzucato, M. (2013). The Entrepreneurial State: Debunking the Public vs. Private Myth in Risk and Innovation. Anthem Press.

National Economic Social Council (NESc) (2005, mayo). The Developmental Welfare State. Núm. 113. Dublín: National Economic \& Social Development Office.

Naciones Unidas (NU)/Comisión Económica para América Latina (CEPAL)/División de Comercio Internacional e Integración (2012a). La República Popular China y América Latina y el Caribe: diálogo y cooperación ante los nuevos desafios de la economía global, Rosales V., O. \& Herreros, S. (Eds.). Santiago de Chile: CEPAL.

Naciones Unidas (NU)/Comisión Económica para América Latina (CEPAL)/División de Comercio Internacional e Integración (2012b). China y América Latina y el Caribe: hacia una relación económica y comercial estratégica. Santiago de Chile: CEPAL.

Ostrom, E. (2010, junio). Beyond Markets and States: Polycentric Governance of Complex Economic Systems. American Economic Review, 100(3), 641-72. 
Ostrom, E. (1996, junio). Crossing the Great Divide: Coproduction, Synergy, and Development. World Development, 24(6), 1073-1087.

Ostrom, E. (1991). Governing the Commons: The Evolution of Institutions for Collective Action. Nueva York: Cambridge University Press

Oszlak, O. \& Gantman, E. (2007). La agenda estatal y sus tensiones. Nordic Journal of Latin American Studies, XXXVII(1).

Panizza, F. (2009). Contemporary Latin America: Development and Democracy beyond the Washington Consensus. Londres: Zed Books.

Piketty, T. (2014). El capital en el siglo XXI. México: Fondo de Cultura Económica.

Puchet Anyul, M., Rojas, M., Salazar, R., Valenti, G. \& Valdés Ugarte, F. (2012). América Latina en los albores del Siglo XXI. 1. Aspectos económicos. México: Flacso México.

Ringen, S., Kwon, H. J., Ilcheong, Y., Taekyoon, K. \& Lee, J. (2011). The Korean State and Social Policy: How South Korea Lifted Itself from Poverty and Dictatorship to Affluence and Democracy. Nueva York: Oxford University Press.

Roberts, K. (2008). ‘¿Es Posible una Socialdemocracia en America Latina?’ ['Is Social Democracy Possible in Latin America?']. Nueva Sociedad, (217), 86-98.

Rosales, V. O. (2013). El dinamismo de China y Asia emergente: oportunidades y desafíos para América Latina y el Caribe. Santiago de Chile: Universidad Miguel de Cervantes/Instituto Latinoamericano de Relaciones Internacionales/JC Sáez Editor.

Ruckert, A. \& MacDonald, L. (2010). 'The Post Neoliberal Mix: New State Practices in Latin America's Big Three'. Unpublished paper. Recuperado de http://www.yorku.ca/robarts/ projects/canadawatch/turning_point/pdfs/Macdonald_Rucke.

Sabel, Ch. \& Zeitlin, J. (2012). Experimentalist Governance. En Levi-Faur, D. (Ed.). The Oxford Handbook of Governance. Oxford, United Kingdom: Oxford University Press.

Sabel, Ch. (2012, diciembre). Dewey, Democracy, and Democratic Experimentalism. Contemporary Pragmatism, 9(2), 35-55.

Schmidt, V. A. \& Thatcher, M. (2013). Resilient Liberalism in Europe's Political Economy. New York: Cambridge University Press.

Sen, A. (2002). Desarrollo y libertad. Madrid: Planeta. 
Silva, E. (2009). Challenging Neoliberalism in Latin America. Nueva York: Cambridge University Press.

Stiglitz, J. (1999). More Instruments and Broader Goals: Moving Toward the Post-Washington Consensus. En Kochendorfer-Lucius, G. \& Pleskovic, B. (Eds.). Development Issues in the 21st Century. Berlín: German Foundation for International Development.

Sokoloff, K. \& Zolt, E. (2006). Inequality and Taxation: Evidence from the Americas on How Inequality May Influence Tax Institutions. Tax Law Review 59(2), 167.

Stubbs, R. (2011). The East Asian Developmental State and the Great Recession: Evolving Contesting Coalitions. Contemporary Politics, 17(2), 151-166.

unctad (2011). Report of the Secretary-General of unctad to unctad XIII. Development-Led Globalization: Towards Sustainable and Inclusive Development Paths. Nueva York: United Nations.

Wade, R. (2010). After the Crisis: Industrial Policy and the Developmental State in Low-Income Countries. Global Policy, (1), 150-161.

Wade, R. (2005). Bringing the State Back In: Lessons from East Asia's Development Experience. Internationale Poltik und Gesellschaft/International Politics and Society (IPG).

Wade, R. (1990). Governing the Market: Economic Theory and the Role of the Government in the East Asian Industrialization. Princeton: Princeton University Press.

Williamson, J. (1990). Latin American Adjustment: How Much Has Happened? Washington, D.C.: Institute for International Economics.

Woo-Cummings, M. (1999). The Development State. Ithaca, N.Y.: Cornell University Press.

World Bank (2008). Governance, Growth, and Development Decision-making. Washington, D. C.

World Economic Forum (2013). The Global Competitiveness Report 2012-2013. Génova.

Zurbriggen, C. (2007). La "falacia tecnocrática” y la reforma del Estado. Revista Nueva Sociedad, 210, 156-172.

Recibido el 29 de noviembre de 2013. Aceptado el 28 de octubre de 2014. 